\section{Myocardial Blood Flow and Myocardial Flow Reserve After Cardiac Transplantation: Mistakes in Diagnostic Value and Prognosis}

TO THE EDITOR: I read with great interest the recent article by Miller et al. published in The Journal of Nuclear Medicine (1). Cardiac allograft vasculopathy (CAV) is a major cause of graft failure after cardiac transplantation. CAV is characterized by diffuse involvement of epicardial coronary arteries and the microvasculature. PET allows quantification of absolute myocardial blood flow (MBF) and myocardial flow reserve (MFR), which may be accurate markers of CAV severity. The authors aimed to compare the diagnostic and prognostic utility of stress MBF and MFR after cardiac transplantation. The diagnostic accuracy for significant CAV (grade 2/3) and prognostic accuracy of stress MBF and MFR, corrected and uncorrected for rate pressure product, were compared. They reported that higher MFR (adjusted hazard ratio, $0.30 ; P<0.001$ ), but not stress MBF (adjusted hazard ratio, 1.14; $P=0$. 656), was associated with reduced all-cause mortality. Preserved MFR $(>2.0)$ identified relatively low-risk patients (annual mortality $4.7 \%$ ) whereas the presence of left ventricular ejection fraction less than $45 \%$ and MFR less than 1.7 identified high-risk patients (annual mortality 51.6\%).

Although this article has provided valuable information, there are some substantial points needing consideration to help the clarity of the method and ensure an accurate interpretation of the study. First, to evaluate diagnostic value, reliability (precision) as a different methodologic issue compared with validity (accuracy) should also be assessed. In this case, application of either weighted or Fliess $\kappa$ is suggested. Without assessing reliability (precision), we cannot talk about the diagnostic value of a test (2-9). Second, it should be noted that, due to the limitation of reported values for accuracy (e.g., sensitivity and specificity are generally used for public health purposes and limited in clinical practice; positive predictive value depends on the prevalence of the outcome), other validity estimates such as likelihood ratios should also be considered. These estimates are more appropriate for advice about accuracy of a diagnostic test for clinical purposes. Thus, reported estimates as in this study can be acceptable; however, when the rest of validity estimates are considered, our final decision can be changed (2-9).

Third, the receiver-operating-characteristic curve is usually used to assess diagnostic accuracy (discrimination) of a diagnostic model. However, for clinical purposes, reporting diagnostic added value of a test is crucially important. The reason is all validity estimates can be acceptable, but diagnostic added value may be negligible. Lastly, for prognosis, assessing internal and external validity is recommended. That is why we need 2 different cohort datasets $(10,11)$. It would be reasonable to assess interaction between predictors before any judgment about prognosis.

In the light of the mentioned points, any conclusion in diagnostic value and prognosis needs to be supported by the methodologic and statistical issues mentioned above. Otherwise, misinterpretation cannot be avoided. So, there is not sufficient evidence to conclude

COPYRIGHT @ 2020 by the Society of Nuclear Medicine and Molecular Imaging. that quantitative PET analysis, and particularly MFR, has diagnostic and prognostic utility.

\section{REFERENCES}

1. Miller RJH, Manabe O, Tamarappoo BK, et al. Comparative prognostic and diagnostic value of myocardial blood flow and myocardial flow reserve after cardiac transplantation. J Nucl Med. 2020;61:249-255.

2. Grobbee DE, Hoes AW. Clinical Epidemiology: Principles, Methods, and Applications for Clinical Research. 2nd ed. Burlington, MA: Jones and Bartlett Publisher; 2015.

3. Sabour S. A quantitative assessment of the accuracy and reliability of o-arm images for deep brain stimulation surgery. Neurosurgery. 2013;72:E696.

4. Sabour S, Ghassemi F. Accuracy, validity, and reliability of the infrared optical head tracker (IOHT). Invest Ophthalmol Vis Sci. 2012;53:4776.

5. Sabour S. Reproducibility of semi-automatic coronary plaque quantification in coronary CT angiography with sub-mSv radiation dose; common mistakes. J Cardiovasc Comput Tomogr. 2016;10:e21-e22.

6. Sabour S. Reproducibility of the external surface position in left-breast DIBH radiotherapy with spirometer-based monitoring: Methodological mistake. J Appl Clin Med Phys. 2014:8; 15:4909

7. Sabour S. Reliability of the ASA physical status scale in clinical practice: Methodological issues. Br J Anaesth. 2015;114:162-163.

8. Sabour S. Validity and reliability of the new Canadian Nutrition Screening Tool in the 'real-world' hospital setting: methodological issues. Eur J Clin Nutr. 2015;69:864.

9. Sabour S, Ghassemi F. The validity and reliability of a signal impact assessment tool: statistical issue to avoid misinterpretation. Pharmacoepidemiol Drug Saf. 2016;25:1215-1216.

10. Sabour S. Prediction of 3-dimensional pharyngeal airway changes after orthognathic surgery: a methodological issue. Am J Orthod Dentofacial Orthop. 2015;147:8.

11. Sabour S. Obesity predictors in people with chronic spinal cord injury: common mistake. J Res Med Sci. 2013;18:1118.

\section{Siamak Sabour \\ Shahid Beheshti University of Medical Sciences Tehran, I.R. Iran, PC: 198353-5511 E-mail: s.sabour@sbmu.ac.ir}

Published online Oct. 4, 2019.

DOI: 10.2967/jnumed.119.236018

\section{Clarifying the Utility of Myocardial Blood Flow and Myocardial Flow Reserve After Cardiac Transplantation}

REPLY: We would like to thank Dr. Sabour for taking an interest in our article, which highlights the potential diagnostic and prognostic utility of PET in patients after cardiac transplantation with known or suspected cardiac allograft vasculopathy (CAV) (1). As discussed by Dr. Sabour (2), there are additional test characteristics that need to be considered before routine implementation of a diagnostic test into clinical practice.

Several previous studies have established the precision and accuracy of PET myocardial blood flow (MBF) measurements (3-10). MBF 